\title{
Review
}

\section{Current perspectives on autoimmune pancreatitis and IgG4-related disease}

\author{
By Kazuichi OKAZAKI ${ }^{* 1, \dagger}$ and Kazushige UCHIDA ${ }^{* 1}$
}

(Communicated by Hiroo ImURA, M.J.A.)

\begin{abstract}
IgG4-related disease (IgG4-RD) is a fibroinflammatory disorder recognized as a novel clinical entity with either synchronous or metachronous multi-organ involvement. Patients with IgG4-RD show diffuse or focal organ enlargement and mass-forming or nodular/thickened lesions with abundant infiltration of IgG4-positive plasmacytes and fibrosis, and such patients respond well to steroid treatment. It should be differentiated from mimics by a combination of serum IgG4 level, imaging features, and histopathological findings. The current first-line drug is corticosteroids, or rituximab in high-risk patients for steroid intolerance. Although relapse rates are high, standardized protocols for relapsed cases have not been approved yet. Based on genetic factors, disease-specific or -related antigens, abnormal innate and adaptive immunity may be involved, although the precise pathogenic mechanism and long-term outcome still remain unclear.
\end{abstract}

Keywords: IgG4-related disease, IgG4, autoimmune pancreatitis

\section{Development process of concepts of autoimmune pancreatitis and IgG4-related disease: before and after the discovery of IgG4}

IgG4-related disease (IgG4-RD) is a fibroinflammatory disorder recognized as a novel clinical entity with multi-organ involvement and unknown origin, associated with abundant infiltration of IgG4-positive cells and fibrosis. ${ }^{1)-3)}$ Involvement of the central nervous system, thyroid, lacrimal glands, salivary glands, breast, lung, liver, bile duct, pancreas, gastrointestinal tract, kidney, prostate, retro-

\footnotetext{
*1 Department of Gastroenterology and Hepatology, Kansai Medical University, Osaka, Japan.

$\dagger \quad$ Correspondence should be addressed: K. Okazaki, Department of Gastroenterology and Hepatology, Kansai Medical University, 2-5-1 Shin-machi, Hirakata, Osaka 573-1010, Japan (e-mail: okazaki@hirakata.kmu.ac.jp).

Abbreviations: AIP: autoimmune pancreatitis; CA: carbonic anhydrase; CDC: comprehensive diagnostic criteria; GEL: granulocytic epithelial lesion; ICDC: International Consensus Diagnostic Criteria; IDCP: idiopathic duct-centric pancreatitis IgG4-RD: IgG4-related disease; IgG4-SC: IgG4-related sclerosing cholangitis; LPSP: lymphoplasmacytic sclerosing pancreatitis; MD: Mikulicz's disease; MOLPS: multiorgan lymphoproliferative syndrome; OOI: other organ involvement; PSC: primary sclerosing cholangitis; ROC: receiver operating characteristic; SIPS: systemic IgG4-related plasmacytic syndrome; SjS: Sjögren's syndrome; TLR: Toll-like receptor; Treg: regulatory $\mathrm{T}$ cell.
}

peritoneum, arteries, lymph nodes, and skin, and good responses to steroid treatment have been reported. ${ }^{1)-3)}$ However, before establishment of the concept of IgG4-RD, each organ lesion has been described independently. ${ }^{1)}$

In 1892, Mikulicz et al. first observed a patient with symmetrical swelling of the lachrymal, parotid and submandibular glands, with massive infiltration of mononuclear cells, and the condition was called Mikulicz's disease (MD); however, it has been classified as an atypical type of Sjögren's syndrome (SjS), which also presents with bilateral, painless, and symmetrical swelling of the lacrimal, parotid, and submandibular glands. Küttner reported a tumor-like enlargement of the submandibular gland of unknown origin that was sometimes a result of stones in the Wharton duct. In 1961, Sarles et al. first observed a case of particular pancreatitis with hypergammaglobulinemia, a prototype of autoimmune pancreatitis (AIP). ${ }^{1}$ In 1991, Kawaguchi et al. proposed a histopathological description of lymphoplasmacytic sclerosing pancreatitis (LPSP) from the resected pancreas of tumor-like appearing pancreatitis, which are clinically difficult to distinguish from pancreatic cancer, and is now regarded as a characteristic histopathological finding of IgG4-related AIP (type 1 AIP). ${ }^{4)}$ In 1995, Yoshida et al. first 
proposed the concept of AIP. ${ }^{5)}$ In 1967, Comings et al. $\left.{ }^{6}\right)$ reported the first familiar case of multifocal fibrosclerosis with retroperitoneal fibrosis, mediastinal fibrosis, sclerosing cholangitis, Riedel's thyroiditis, and pseudotumor of the orbit, which is now regarded as the syndrome of IgG4-RD. ${ }^{1-3)}$

In 2001, Hamano et al. reported increased serum levels of IgG4 in Japanese patients with AIP, ${ }^{7)}$ an epoch-making discovery in the history of IgG4-RD. Thereafter, many studies of AIP have been reported, mainly by Japanese investigators. The histopathological findings of LPSP are characterized by the periductal localization of predominantly CD4 positive T-cells, IgG4-positive plasma cells, storiform fibrosis with acinar cell atrophy frequently resulting in stenosis of the main pancreatic duct, and obliterative fibrosis. ${ }^{1)}$ Approximately $60-80 \%$ of patients with AIP show obstructive jaundice with sclerosing cholangitis (IgG4-related sclerosing cholangitis; IgG4-SC) and other organ involvements (OOIs), in which cholangiographic features are similar to those of primary sclerosing cholangitis (PSC), pancreatic cancer, or cholangiocarcinoma. The steroid responses and the prognoses of sclerosing cholangitis associated with AIP differ from patients with PSC, which suggests different pathological conditions. In 2006, Kamisawa et al. ${ }^{8)}$ suggested a concept of a systemic sclerosing disease with fibrosis and abundant infiltration of IgG4-positive plasma cells based on multifocal fibrosclerosis proposed by Comings et al. Further histological and clinical profiling of patients with "AIP" reveals two distinct subtypes, type 1 and type $2 .{ }^{9)}$ Type 1 AIP is now regarded as a pancreatic manifestation of IgG4-RD, and type 2 AIP is supposed to be a distinctive pancreatic disease with granulocytic epithelial lesion (GEL) and occasional association with ulcerative colitis. $^{9)}$

Conversely, most patients with MD show elevated serum levels of IgG4, negative anti-SS-A/ Ro or anti-SS-B/La antibodies, infiltration of IgG4positive plasma cells into the glands, and recovery of secretion with steroid treatment. Patients with MD often show steroid-responsive OOIs such as AIP, sclerosing cholangitis, retroperitoneal fibrosis, enlarged celiac and hilar lymph nodes, chronic thyroiditis, or interstitial nephritis, which suggests that MD is completely different from Sjögren's syndrome. ${ }^{1)-3)}$

In addition to the original concept of multifocal idiopathic fibrosclerosis, recent studies led us to develop a novel concept of a systemic disease such as IgG4-related systemic sclerosing disease, ${ }^{8)}$ systemic IgG4-related plasmacytic syndrome (SIPS), ${ }^{10)}$ or IgG4-positive multiorgan lymphoproliferative syndrome (IgG4-MOLPS), ${ }^{11)}$ all of which may refer to the same conditions. Based on these findings, the Japanese Research Committees for "Systemic IgG4related Sclerosing Disease" (chaired by Okazaki, K.) and "IgG4-MOLPS" (chaired by Umehara, M.) supported by the Research for Intractable Disease program from the Ministry of Health, Labour, and Welfare of Japan, have proposed the comprehensive term" ${ }^{2)}$ and diagnostic criteria for "IgG4-related disease (IgG4-RD)". ${ }^{12)}$ The first International Symposium on IgG4-RD held in Boston (chaired by Stone, J.) endorsed the Japanese concept and proposed nomenclatures and pathological criteria for individual organ lesions. ${ }^{3), 13)}$

\section{Current concepts of IgG4-RD}

Patients with IgG4-RD, either synchronously or metachronously, show diffuse or focal organ enlargement and mass-forming or nodular/thickened lesions in various organs with abundant infiltration of IgG4-positive plasmacytes with fibrosis. ${ }^{1-3)}$ IgG4$\mathrm{RD}$ includes a wide variety of diseases, including MD, AIP, hypophysitis, Riedel thyroiditis, interstitial pneumonitis, interstitial nephritis, prostatitis, lymphadenopathy, retroperitoneal fibrosis, inflammatory aortic aneurysm, and inflammatory pseudotumor (Fig. 1). Approximately 10-20\% of the patients have a single organ involvement. Although it is unclear whether the pathogenic mechanism is the same among individual organs or not, recent studies have suggested possible multi-pathogenic factors in the development of AIP similar to other immunogenic diseases. Based on genetic factors, disease-specific or -related antigens, abnormal innate and adaptive immunity may be involved. IgG4-RD mainly affects middle-aged to elderly men except for $\mathrm{MD}$, in which previous epidemiological studies did not show a gender difference. Clinical symptoms vary depending on the individual organs involved, but these are dramatically relieved by steroid therapy in many cases $;^{1-3)}$ however, long-term prognosis still remains unclear. Some patients develop serious complications such as obstructive jaundice due to hepatic, gallbladder, or pancreatic disease; hydronephrosis due to retroperitoneal fibrosis; or respiratory symptoms due to pulmonary disease. The infiltration of IgG4-positive cells, increased serum levels of IgG4, storiform fibrosis and obliterative phlebitis are characteristic in most organ involve- 


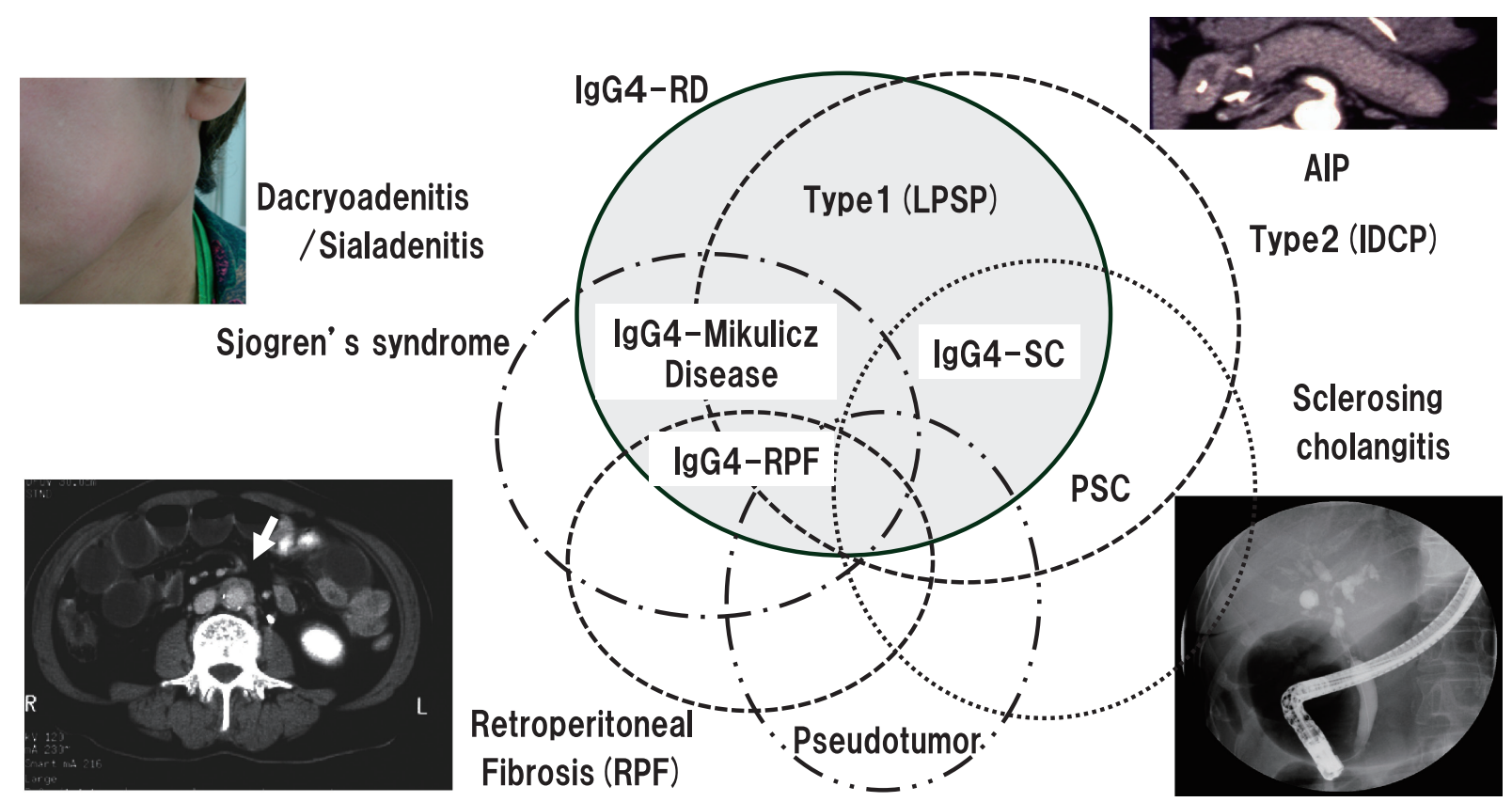

Fig. 1. Current concept of IgG4-related disease (cited from ref. 8 with permission). IgG4-RD contains multi-organ involvement simultaneously or metachronously.

Table 1. Comprehensive diagnostic criteria for IgG4-related disease, 2011 (cited from ref. 12 with permission)

1. Clinical examination showing characteristic diffuse/localized swelling or masses in single or multiple organs

2. Hematological examination shows elevated serum IgG4 concentrations $(135 \mathrm{mg} / \mathrm{dL})$

3. Histopathologic examination shows:

(1) Marked lymphocyte and plasmacyte infiltration and fibrosis.

(2) Infiltration of IgG4+ plasma cells: ratio of IgG4+/IgG+ cells $>40 \%$ and $>10$ IgG4+ plasma cells/HPF.

Definite: 1$)+2)+3$ Probable: 1$)+3$ ) Possible: 1$)+2$ )

However, it is important to differentiate IgG4-RD from malignant tumors of each organ (e.g., cancer, lymphoma) and similar diseases (e.g., Sjögren's syndrome, primary sclerosing cholangitis, Castleman's disease, secondary retroperitoneal fibrosis, Wegener's granulomatosis, sarcoidosis, and Churg-Strauss syndrome) by additional histopathological examination. Even when patients cannot be diagnosed using the CCD criteria, they may be diagnosed using organ-specific diagnostic criteria for IgG4-RD.

ments of IgG4-RD including pancreatic, biliary tract, retroperitoneal, and renal lesions, but storiform fibrosis and obliterative phlebitis are rarely observed in lymph nodes, salivary, or lacrimal glands.

In the International Symposium on IgG4-RD, the nomenclature of individual organ manifestations of IgG4-RD were proposed (Table 1) using "IgG4related" as a modifier, except for the pancreatic manifestation. ${ }^{3)}$ Formerly called Mikulicz's disease, Riedel thyroiditis, or Küttner tumor, Ormand's disease is replaced by IgG4-related dacryoadenitis and sialoadenitis, IgG4-related thyroid disease, IgG4- related submandibular gland disease, and IgG4related retroperitoneal fibrosis, respectively. The pancreatic manifestation of IgG4-RD is termed "type 1 autoimmune pancreatitis (IgG4-related pancreatitis)", because it is widely accepted among gastroenterologists and pancreatic surgeons, and helps to discriminate between type 1 and type 2 AIP, which are not a part of the IgG4-RD spectrum. When the pathogenesis of type 2 AIP is clarified, the term "type 1 AIP" may be replaced by "IgG4-related pancreatitis". 3) 


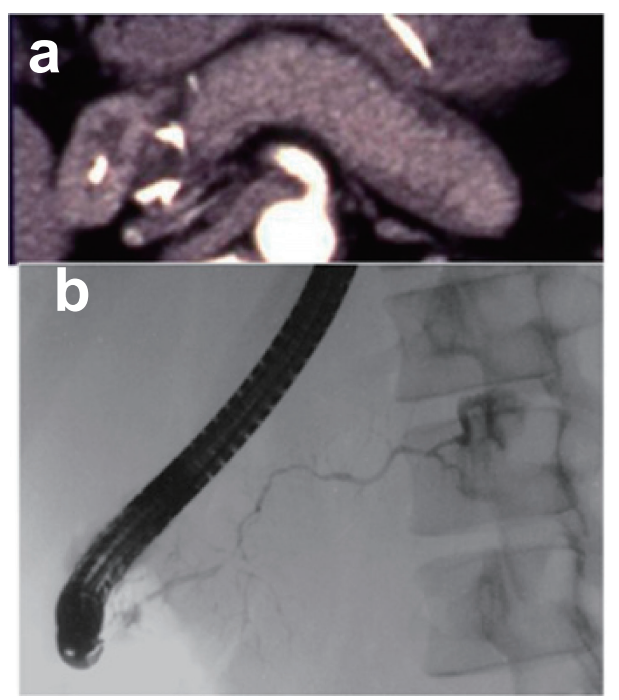

\section{C}

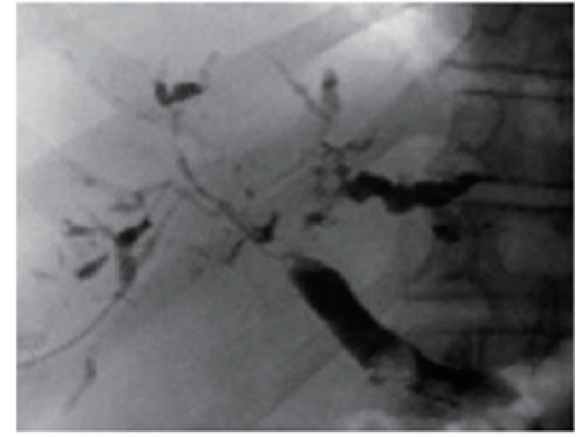

Fig. 2. Pancreatic images of type 1 AIP (cited from ref. 1 with permission). a. Dynamic CT shows diffuse swelling of the pancreas with late phase enhancement and low-density rim. b. Endoscopic pancreatography shows diffusely irregular narrowing of the main pancreatic duct. c. Endoscopic cholangiography shows beaded like and long stenotic biliary duct similar to primary sclerosing cholangitis.

\section{Current concepts of AIP: type 1 AIP (IgG4- related pancreatitis) and type 2 AIP}

Recent studies have suggested that AIP is classified as two distinct subtypes, types 1 and type 2, and the International Consensus Diagnostic Criteria (ICDC) for AIP, which enabled the differentiation of type 1 from type 2 AIP and is the most widely accepted major diagnostic criteria. ${ }^{9)}$ Clinically, type 1 AIP (IgG4-related pancreatitis) seems to be the pancreatic manifestation of IgG4-RD, characterized by: (i) mild abdominal symptoms, usually without acute attacks of pancreatitis; (ii) occasional occurrence of obstructive jaundice; (iii) increased serum gammaglobulin, IgG, and/or IgG4 concentrations; (iv) presence of autoantibodies; (v) diffuse, segmental, or focal enlargement of the pancreas with a capsule-like low-density rim on dynamic computed tomography/magnetic resonance imaging (CT/MRI) images; (vi) irregular narrowing of the main pancreatic duct on endoscopic retrograde cholangiopancreatography images; (vii) histopathologically, LPSP: abundant infiltration of lymphocytes and IgG4-positive plasmacytes and fibrosis, and obliterative phlebitis; and (viii) occasional association with OOIs (Figs. 2 and 3). Patients with type 1 AIP often have obstructive jaundice, with both pancreatic and extrapancreatic manifestations responding to steroid therapy. ${ }^{1,8), 9)}$
Histological examination by American and European pathologists of the resected pancreases of patients with chronic non-alcoholic pancreatitis revealed another histopathological pattern, called idiopathic duct-centric pancreatitis (IDCP) $)^{14)}$ or AIP with GELs ${ }^{15)}$ later named as type 2 AIP. ${ }^{9)}$ Although patients with type 2 AIP show similar pancreas images to type 1 AIP, they show different clinicopathological features from type 1 AIP such as no elevation of serum IgG4, occasional infiltration of IgG4-positive plasma cells, no autoantibodies, or no involvement of other organs, except for inflammatory bowel disease (Figs. 4 and 5). The most characteristic feature of type 2 AIP is GEL, commonly accompanied by destruction and obliteration of the pancreatic duct. ${ }^{9)}$ Although type 1 AIP (LPSP type) often occurs in older men and is accompanied by a variety of extrapancreatic lesions, type 2 AIP (IDCP/GEL type), has no gender difference, younger age at onset (often $<40$ years), and is frequently associated with inflammatory bowel disease (about 30\%). Thus, after a worldwide debate over the diagnostic criteria for AIP, LPSP has been defined as type 1 (IgG4-related pancreatitis) and IDCP/AIP with GEL has been defined as type $2 .{ }^{9)}$

\section{Diagnosis of IgG4-related disease}

Patients with IgG4-related disease show organ enlargement or nodular/hyperplastic lesions in or- 

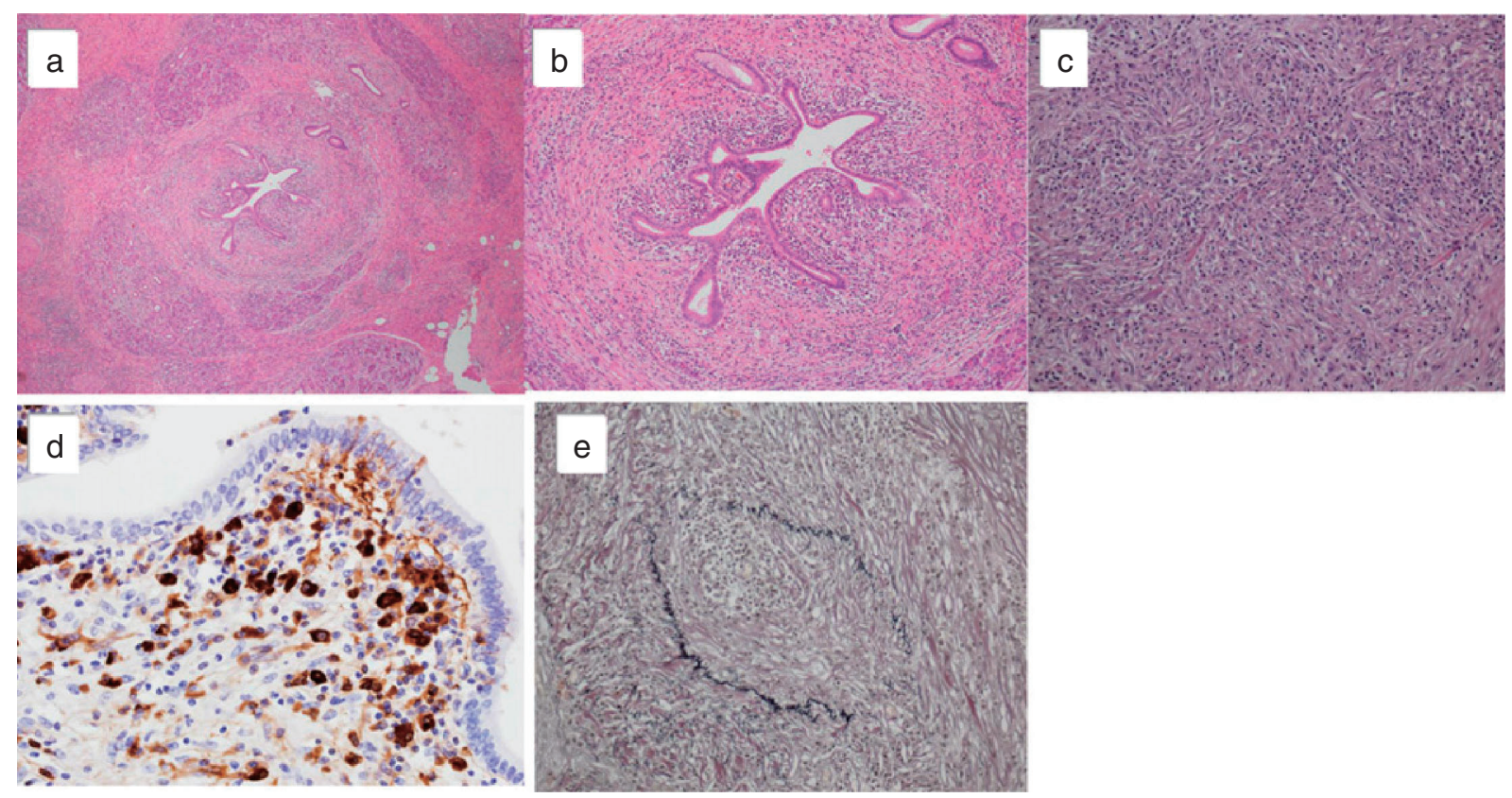

Fig. 3. Lymphoplasmacytic sclerosing pancreatitis (LPSP) (cited from ref. 1 with permission). a. Abundant infiltration of plasma cells and lymphocytes around the pancreatic duct with fibrosis $(\mathrm{H} \& \mathrm{E}, \times 40)$. b. Abundant infiltration of plasma cells and lymphocytes around the pancreatic duct with fibrosis $(\mathrm{H} \& \mathrm{E}, \times 200)$. c. Storiform fibrosis $(\mathrm{H} \& \mathrm{E}, \times 400)$. d. Abundant infiltration of IgG4 positive plasma cells anti-IgG4 antibody staining $(\mathrm{H} \& \mathrm{E}, \times 400)$. e. Obliterative phlebitis (Evans staining, $\times 400)$.

gans in the entire body, synchronously or metachronously, due to the prominent infiltration and fibrosis of lymphocytes and plasmacytes. The organs known to be affected include the central nervous system, lacrimal/salivary glands, thyroid gland, lungs, pancreas, biliary duct, liver, gastrointestinal tracts, kidneys, prostate gland, retroperitoneum, skin, arteries, and lymph nodes. Clinical symptoms vary depending on the organ in which the lesions are located, which suggests that it is hard to establish criteria covering all patients with IgG4-RD. Therefore, some specific diagnostic criteria have been proposed for each involved organ, such as for IgG4related Mikulicz's disease (IgG4-related dacryoadenitis/sialadenitis), ${ }^{12), 16)}$ type 1 AIP (IgG4-related pancreatitis), ${ }^{9)}$ and IgG4-related kidney disease. ${ }^{17)}$ However, these organ specific criteria do not cover other organs or are not familiar to general clinicians and non-specialists. Therefore, Japanese investigators have proposed comprehensive diagnostic criteria (CDC) for IgG4-RD, containing three major criteria (clinical, hematological, and histopathological examinations) for practical use by general physicians and non-specialists (Table 2). ${ }^{12}$ )

Clinical examination. Physical examinations and imaging by ultrasound/CT/MRI can show the characteristic diffuse/localized swelling, masses, or thickness in single or multiple organs.

Hematological examination. The cutoff value for serum IgG4 concentration, $135 \mathrm{mg} / \mathrm{dL}$, was based on receiver operating characteristic (ROC) curves, ${ }^{7)}$ and its validity was confirmed. ${ }^{18)}$ In patients with single-organ involvement and serum IgG4 concentration $<135 \mathrm{mg} / \mathrm{dL}$, the $\mathrm{IgG} 4 / \mathrm{IgG}$ ratio may be helpful in making a diagnosis. However, elevated IgG4 may also be observed in other diseases (e.g., atopic dermatitis, pemphigus, asthma, and multicentric Castleman's disease), and especially in about $10 \%$ of malignancies, which suggests that high serum IgG4 is not necessarily a specific marker of IgG4RD. ${ }^{18)}$ Therefore, at present, the significance of elevated IgG4 in the pathogenesis/pathophysiology of IgG4-RD still remains unknown.

Histopathologic examination. Although tissue biopsies are difficult to obtain from some organs, including the pancreas, retroperitoneum, and ocular cavity, histopathological examination is important. Marked lymphocyte and plasmacyte infiltration with storiform fibrosis or obliterative phlebitis is characteristic of IgG4-RD. IgG4/IgG-positive cells $>40 \%$ have been reported in lymph nodes of patients with IgG4-RD. On the other hand, $>10$ 

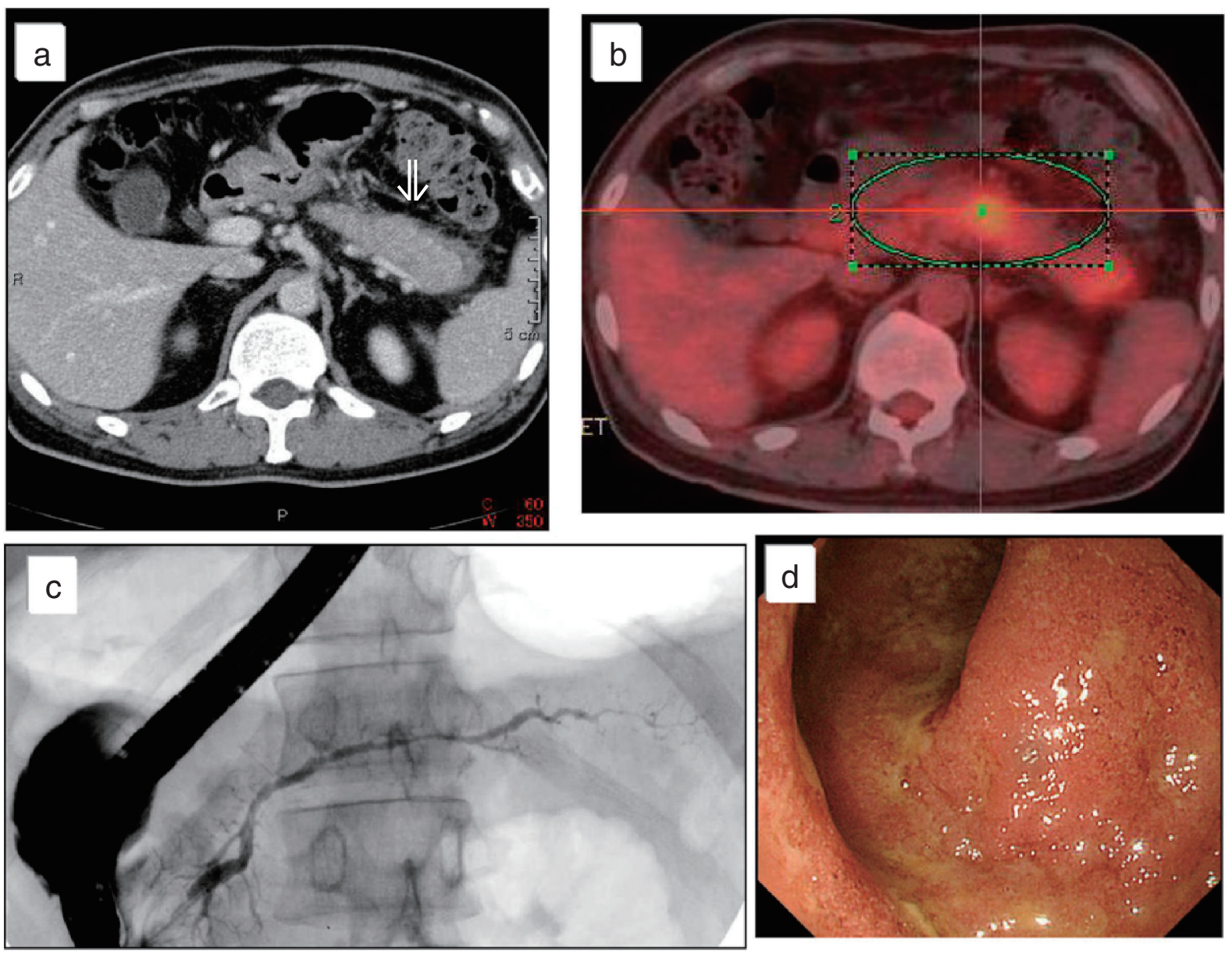

Fig. 4. Pancreatic images of type 2 AIP (cited from ref. 1 with permission). a. Dynamic CT shows diffuse swelling of the pancreas with late-phase enhancement and low-density rim (arrow) similar to type 1 AIP. b. Positron emission tomography-computed tomography (PET-CT) shows diffusely abnormal accumulation of FDG. c. Endoscopic pancreatography shows irregular narrowing of the main pancreatic duct. $\mathbf{d}$. Colonoscopy shows proctitis type of ulcerative colitis.
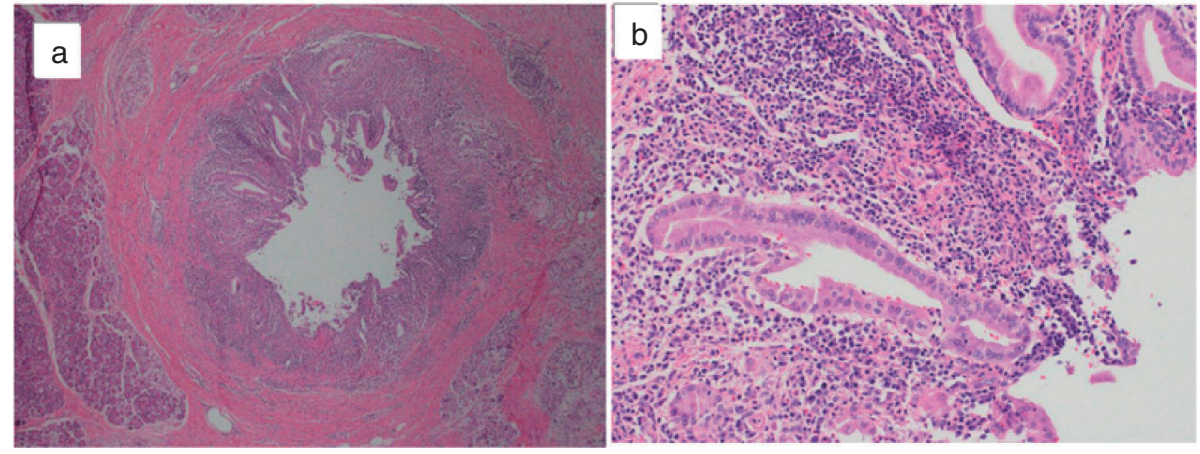

Fig. 5. Granulocytic epithelial lesion (cited from ref. 1 with permission). a. Abundant infiltration of granulocytes into and around medium-sized pancreatic duct with destruction of epithelia $(\mathrm{H} \& \mathrm{E}, \times 40)$. b. Abundant infiltration of granulocytes into and around medium-sized pancreatic duct with destruction of epithelia $(\mathrm{H} \& \mathrm{E}, \times 200)$. 
Table 2. Preferred nomenclature for individual organ manifestations of IgG4-related disease (cited from ref. 3 with permission)

\begin{tabular}{|c|c|}
\hline Organ System/Tissue & Preferred Name \\
\hline Pancreas & Type 1 autoimmune pancreatitis (IgG4-related pancreatitis) \\
\hline Eye & $\begin{array}{l}\text { IgG4-related ophthalmic disease is the general term for the peri-ocular manifestations } \\
\text { of this disease. There are several subsets, outlined below. }\end{array}$ \\
\hline Lacrimal glands & IgG4-related dacryoadenitis \\
\hline $\begin{array}{l}\text { Orbital soft tissue (orbital inflammatory } \\
\text { pseudotumor) }\end{array}$ & IgG4-related orbital inflammation \\
\hline Extra-ocular muscle disease & IgG4-related orbital myositis \\
\hline $\begin{array}{l}\text { Orbit with involvement of multiple anatomic } \\
\text { structures }\end{array}$ & $\begin{array}{l}\text { IgG4-related pan-orbital inflammation (includes lacrimal gland disease, extra-ocular } \\
\text { muscle involvement, and other potential intra-orbital complications) }\end{array}$ \\
\hline $\begin{array}{l}\text { Salivary glands (parotid and submandibular } \\
\text { glands) }\end{array}$ & $\begin{array}{l}\text { IgG4-related sialadenitis or, more specifically, IgG4-related parotitis or IgG4-related } \\
\text { submandibular gland disease }\end{array}$ \\
\hline Pachymeninges & IgG4-related pachymeningitis \\
\hline Hypophysis & IgG4-related hypophysitis \\
\hline Thyroid (Riedel's thyroiditis) & IgG4-related thyroid disease \\
\hline Aorta & IgG4-related aortitis/peri-aortitis \\
\hline Arteries & IgG4-related periarteritis \\
\hline Mediastinum & IgG4-related mediastinitis \\
\hline Retroperitoneum & IgG4-related retroperitoneal fibrosis \\
\hline Mesentery & IgG4-related mesenteritis \\
\hline Skin & IgG4-related skin disease \\
\hline Lymph node & IgG4-related lymphadenopathy \\
\hline Bile ducts & IgG4-related sclerosing cholangitis \\
\hline Gallbladder & IgG4-related cholecystitis \\
\hline Liver & $\begin{array}{l}\text { IgG4-related hepatopathy (refers to liver involvement that is distinct from biliary } \\
\text { tract involvement) }\end{array}$ \\
\hline Lung & IgG4-related lung disease \\
\hline Pleura & IgG4-related pleuritis \\
\hline Pericardium & IgG4-related pericarditis \\
\hline Kidney & $\begin{array}{l}\text { IgG4-related kidney disease. The specific renal pattern should be termed IgG4-related } \\
\text { tubulointerstitial nephritis and membranous glomerulonephritis secondary to IgG4- } \\
\text { RD. Involvement of the renal pelvis should be termed IgG4-related renal pyelitis. }\end{array}$ \\
\hline Breast & IgG4-related mastitis \\
\hline Prostate & IgG4-related prostatitis \\
\hline
\end{tabular}

IgG4-positive plasma cells are recommended in the diagnosis of type 1 AIP. ${ }^{9)}$ Based on these findings, the CDC for IgG4-RD recommend both the ratio of IgG4/IgG-positive cells $>40 \%$ and infiltration of $>10$ IgG4-positive plasma cells/HPF for definitive diagnosis. ${ }^{12)}$ Eosinophilic infiltration is often observed along with infiltration of IgG4-positive cells.

Although the sensitivity of the CDC for definitive IgG4-RD is low in organs from which it is difficult to take biopsy specimens, it can detect possible cases of IgG4-RD. In probable or possible cases, organ-specific criteria should be used concurrently.

\section{Treatment}

Although some studies of the treatment for IgG4-RD have been proposed, ${ }^{19), 20)}$ comprehensive management for IgG4-RD has not yet been established. Because the clinical features of patients with IgG4-RD depend on characteristics of the organs involved, the current consensus for the treatment of type $1 \mathrm{AIP},{ }^{21)}$ as a representative lesion of IgG4-RD, is described (Fig. 6).

Induction for remission. For induction of remission, steroids can successfully achieve induction in most patients with type 1 AIP $(\sim 90 \%) .{ }^{21), 22)}$ The 


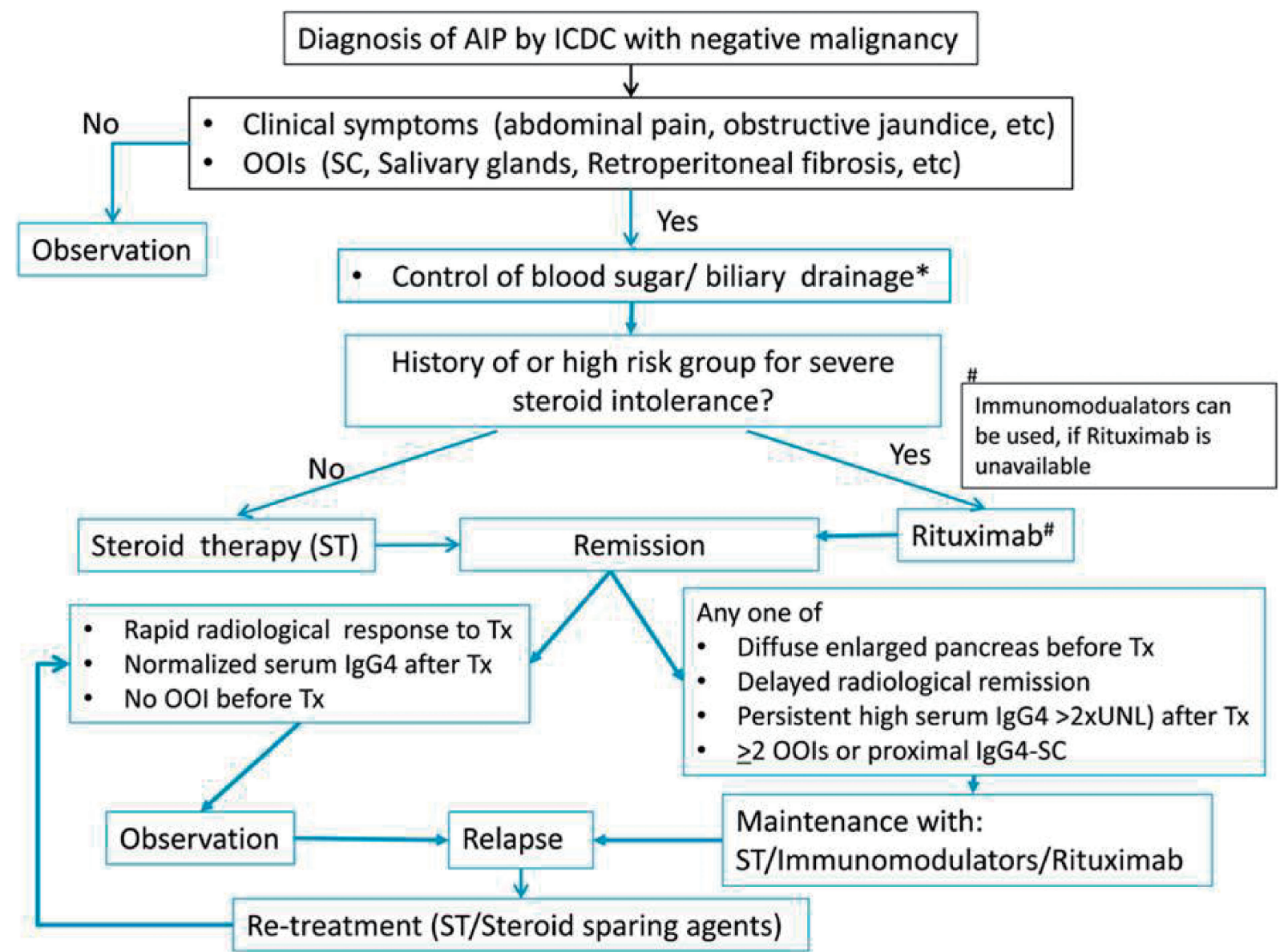

Fig. 6. Therapeutic algorithm for type 1 AIP (cited from ref. 26 with permission). This algorithm is based on Eastern and Western experts' opinions to recommend standard treatment of AIP worldwide. ICDC: International Consensus Diagnostic Criteria for autoimmune pancreatitis. OOI: other organ involvement. ST: steroid treatment. *: In some cases of mild jaundice without signs of infection, steroid treatment alone can be performed safely without biliary drainage.

Japanese guidelines ${ }^{23)}$ and international consensus ${ }^{21)}$ have recommended that steroids are the first-line agent in all active and symptomatic patients with untreated AIP, who have obstructive jaundice or abdominal and/or back pain due to pancreatic or other organ involvements, unless patients have any contraindications or high risks for steroid use. They have also recommended steroid treatment even in asymptomatic patients with persistent pancreatic mass on imaging, or those with associated IgG4related sclerosing cholangitis (IgG4-SC) who have persistent liver test abnormalities. ${ }^{21)}$ In patients with contraindications to steroid treatment, rituximab can induce remission as a single agent. ${ }^{21), 24)}$ Except for rituximab, however, other steroid-sparing agents such as thiopurines are poorly effective as a single agent for the induction of remission. ${ }^{25)}$ Before any initial steroid therapy, management of blood glucose in diabetic patients or biliary drainage in patients with obstructive jaundice are recommended, ${ }^{23)}$ because biliary drainage can prevent biliary infection, and brushing or bile juice cytology can differentiate IgG4-SC from biliary malignancy. In some cases of mild jaundice without signs of infection, however, steroid treatment alone can be performed safely without biliary stenting. ${ }^{26)}$

Relapse and second-line treatment. According to international or Japanese multicenter studies, ${ }^{27)} 30-50 \%$ of the patients with type 1 AIP experienced relapse. Most risk factors for relapsing remain poorly understood and require further study. A history of relapse seems to be a strong risk factor for future relapse. Although an international study ${ }^{22}$ ) could not confirm the finding, Japanese national retrospective $^{27)-29)}$ studies have suggested that diffuse pancreatic enlargement type, and persistently 
high, low decreased rate or re-elevation of serum IgG4 levels after steroid therapy, may be predictors of AIP relapse. Association with IgG4-related SC, especially the proximal type of IgG4-SC seems to be a risk factor for relapse. In addition to serum IgG4 levels, circulating immune complexes have been reported as useful early predictors of relapse. ${ }^{30)}$

Although the usefulness of steroids as an initial treatment is agreed upon, there is no "gold standard" for treatment in relapse cases. The international consensus recommends re-administration or and increased dose of steroid or steroid-sparing agent, such as immunosuppressive agents or rituximab. ${ }^{19)}$

Maintenance treatment. Although there is no high-level evidence regarding maintenance therapy, certain patients benefit from maintenance therapy after a successful course of induction therapy. ${ }^{19), 23), 31)}$ Whereas some patients do not relapse after withdrawal of steroids after remission is achieved, some patients relapse during steroid taper or require relatively high-dose maintenance therapy. Therefore, to determine the indications for maintenance therapy, it is important to evaluate disease activity during induction therapy.

Maintenance therapy may consist of low-dose glucocorticoids or any of the steroid-sparing agents. Retrospective studies from Asian countries, mainly Japan $^{27)}$ and South Korea, ${ }^{32)}$ have suggested that steroid maintenance monotherapy may prevent relapse after remission. In addition to retrospective studies, a recent prospective Japanese multicenter study has shown that steroid maintenance therapy provides beneficial outcomes after remission. ${ }^{33)}$

In cases of low disease activity such as involvement in the pancreas alone with segmental/focal lesions without any OOIs and complete radiological remission with normalized $\operatorname{IgG} / \operatorname{IgG} 4$ in rapid response to steroid, steroids can be tapered off within 3 months followed by no steroid maintenance. ${ }^{21)}$ On the other hand, maintenance therapy using a low dose of steroid, immunomodulators, or rituximab is recommended in patients with type 1 AIP showing diffuse enlargement of the pancreas, delayed radiological remission or persistently high serum IgG4 $(>2 \times$ upper limit of normal [ULN]) after treatment, or $\geq 2$ OOIs, or association with proximal IgG4-SC before treatment. ${ }^{21)}$

\section{Outcome of type 1 AIP and IgG4-RD}

Clinical symptoms vary depending on the individual organs involved and are dramatically relieved by steroid therapy in many cases; ${ }^{19)-21), 23)}$ however, long-term prognosis still remains unclear. Corticosteroids improve pancreatic exocrine and endocrine function by reducing inflammation, fibrosis, and regeneration through correct aberrant cystic fibrosis transmembrane conductance regulator localization in the duct and regenerate acinar cells in AIP; ${ }^{34)}$ therefore, the short-term prognosis of AIP appears to be positive with steroid therapy. On the other hand, it is unclear whether the long-term outcome is positive, because there are many unknown factors, such as relapse, pancreatic exocrine or endocrine dysfunction, and associated malignancy.

Although not commonly observed, approximately $10 \%(7-40 \%)$ of the patients with type 1 AIP may develop pancreatic calcification or chronic pancreatitis. ${ }^{35)-39)}$ Maruyama et al. ${ }^{38), 39)}$ proposed a sequential progression mechanism for the transition of AIP to chronic pancreatitis. They identified two risk factors, pancreatic head swelling and nonnarrowing main pancreatic duct in the pancreatic body, which may cause pancreatic juice stasis in the upstream pancreatic duct and increased intrapancreatic duct pressure that is resistant to typical AIPspecific main pancreatic duct narrowing in the pancreatic body.

Although the association of malignant tumors with type 1 AIP or IgG4-RD is controversial, several cases of pancreatic cancer or other malignancies have been described in patients with AIP or IgG4RD. ${ }^{22), 40)-44)}$ In recent reports, ${ }^{22), 40)-44)}$ in about 1,000 patients, $8.5 \%(10.1-13.9 \%)$ of IgG4-RD cases and $1 \%$ of AIP cases (2.1-13.9\%) exhibited malignant tumors. Therefore, it is noted that pancreatic cancer or other malignant tumors may be complicated with AIP or IgG4-RD. A Japanese multicenter study of 108 cases of AIP reported that the highest risk for cancer is in the first year after AIP diagnosis. The absence of a relapse after successful treatment of the coexisting cancers suggests that AIP may develop as a paraneoplastic syndrome in some patients. ${ }^{42}$ ) Conversely, in an international multicenter study, the most frequently occurring cancers during followup were gastric, lung, and prostate, but not pancreatic, cancer. ${ }^{22)}$ Kamisawa et al. ${ }^{44)}$ investigated K-ras mutations in gallbladder and pancreas tissues obtained from AIP patients, and demonstrated significant K-ras mutation in the pancreatic and biliary regions, which suggested the possibility of AIP as a risk factor for pancreatic and bile duct cancer. 


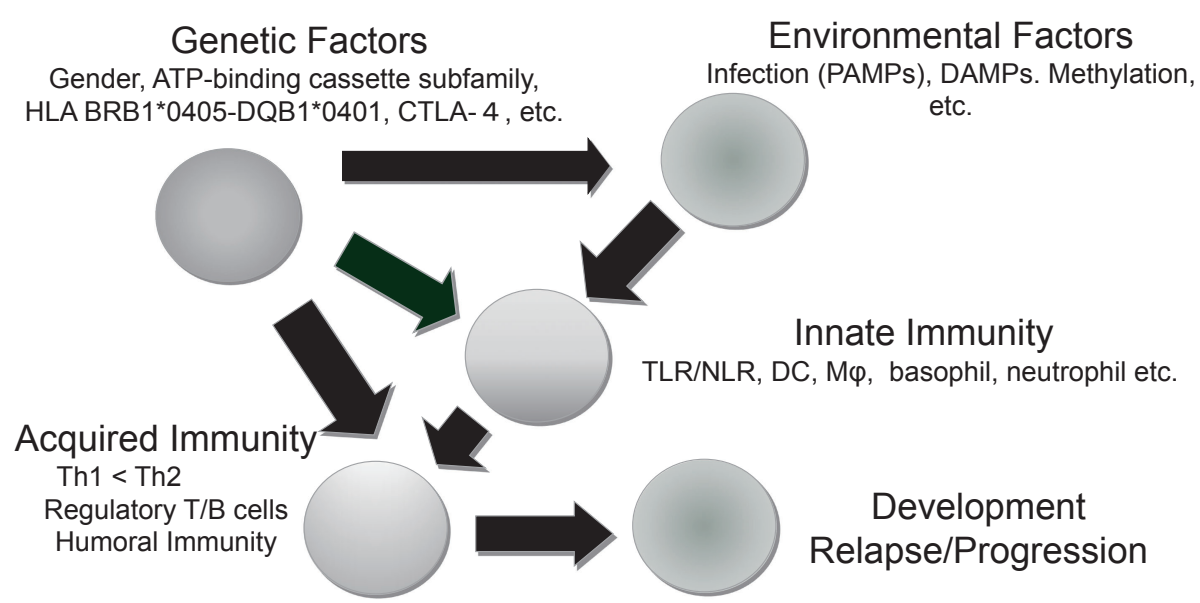

Fig. 7. Multiple pathogenic factors in the development of type 1 AIP (cited from ref. 48 with permission). Based on genetic factors, disease-specific or -related antigens, abnormal innate and adaptive immunity may be involved.

\section{Recent advances in the pathogenic mechanisms of AIP and IgG4-RD}

Although it is unclear whether the pathogenic mechanism is the same among individual organs or not, recent studies have suggested possible multipathogenic factors in the development of AIP similar to other immunogenic diseases. Based on genetic factors, disease-specific or -related antigens, abnormal innate and adaptive immunity may be involved $(\text { Fig. } 7)^{45)}$.

Immunogenic backgrounds. Although immunogenic backgrounds are unknown in IgG4-RD, the susceptibility to AIP in Japanese patients, most of whom exhibit IgG4-RD, may be associated with the class II antigen haplotype of the major histocompatibility complex (HLA-DRB1*0405DQB1*0401) ${ }^{46)}$ polymorphism of nuclear factor- $\kappa \mathrm{B}$ and Fc-receptor-like 3 genes expressed on B cells. ${ }^{47)}$ An inhibitory molecule, cytotoxic $\mathrm{T}$ lymphocyte antigen-4 (CTLA-4; CD152), expressed on the activated memory $\mathrm{T}$ cells or $\mathrm{CD} 4{ }^{+} \mathrm{CD} 25^{+}$regulatory $\mathrm{T}$ cells (Tregs), was independently reported as a susceptibility factor. ${ }^{48), 49)}$ Based on immunogenic backgrounds, abnormal conditions of immune responses may be involved in the development of type 1 AIP, although the precise pathogenic mechanisms remain unclear.

Innate immunity. Recently, abnormal innate immunity has been demonstrated in some patients with IgG4-RD. ${ }^{50)}$ Activation of NOD-2 and Toll-like receptor (TLR) ligands on monocytes or basophils from patients with IgG4-related AIP enhances IgG4 responses via B-cell activating factor and IL-13, although the specific pathogens still remain unclear. ${ }^{50), 51)}$ Moreover, abundant infiltration of TLR7 positive M2-macrophages was observed in pancreatic tissues from type 1 AIP cases. $^{52)}$ In animal models, activation of TLR3 (polyinosinic:polycytidylic acid) or TLR4 (lipopolysaccharide) can induce immune-mediated cholangitis, pancreatitis, and sialadenitis similar to human IgG4-RD. ${ }^{53)}$ Recently, possible roles of basophils, which are activated via TLR signaling, may be involved in the development of type 1 AIP. ${ }^{54}$

Possible roles of IgG4 in IgG4-RD. Although the association of IgE-mediated allergy and IgG4 antibodies is well known, IgG4 characteristics are still poorly understood. IgG4 is involved in an immune process referred to as 'Fab-arm exchange', which involves the swapping of a heavy chain and attached light chain (half-molecule) with a heavy-light chain pair from another molecule. This usually results in asymmetric antibodies with two different antigencombining sites. ${ }^{55)}$ Although these modified antibodies are hetero-bivalent, they behave as monovalent antibodies. Another aspect of IgG4 is that it mimics IgG rheumatoid factor activity by interacting with IgG, namely through Fc-mediated aggregation. ${ }^{56)}$ IgG4 seems to be associated with a pathogenic effect in a few situations. In pemphigus, recognition of skin autoantigens (desmogleins) by IgG4 is at the origin of the disease process. ${ }^{57)}$ The most recent study of structural determinants of human IgG4-Fc using crystallization suggested that $\mathrm{Fc}-\mathrm{Fc}$ interactions are compatible with intact $\mathrm{IgG} 4$ 
molecules and may provide a model for the formation of aggregates of IgG4 that can cause disease pathology in the absence of antigen. ${ }^{58)}$

Another recent study of the regulation of $\operatorname{IgG} 4$ showed that IgG4-RD may reflect an excessive production of anti-inflammatory cytokines such as IL-10, which triggers an overwhelming expansion of IgG4-producing plasma cells. ${ }^{59)-63)}$ Increased peripheral inducible-memory Tregs are positively correlated with serum levels of IgG4. ${ }^{60)}$ In addition, prominent infiltration of Tregs upregulated IL-10 in the liver of patients with IgG4-SC. ${ }^{59)}$ These findings suggested that IgG4 does not act as a pathogenic factor, nor is it an anti-inflammatory factor in IgG4-RD. Further studies are necessary to clarify the precise role of IgG4 in IgG4-RD.

The complement system. Patients in the active stages of AIP occasionally show decreased complement (C3, C4) with elevated circulating immune complex, as well as elevated serum levels of IgG4 and the IgG4 subclass of immune complexes. ${ }^{64)}$ However, a previous study showed that the classical pathway of complement activation through IgG1 may be involved in the development of AIP, as opposed to mannose-binding lectin or alternative pathways through IgG4. ${ }^{64)}$

Autoantibodies and candidate of target antigens. Although some patients with IgG4-RD have non-specific antibodies such as an anti-nuclear antibody, this is rare. From the viewpoint of $\operatorname{IgG} 4$ function, it remains unclear whether IgG4-RD is an autoimmune or an allergic disease. Although diseasespecific targets are unknown, the occasional coexistence of OOIs leads us to consider that there may be common target antigens in the organs involved, especially in the pancreas, which exhibit a high coincidence. Among candidate antigens previously reported, lactoferrin, ${ }^{65), 66)}$ carbonic anhydrase (CA)II, ${ }^{65)-68)} \mathrm{CA}-\mathrm{IV},{ }^{69)}$ and pancreatic secretory trypsin inhibitor, ${ }^{70)}$ are distributed in the pancreas, salivary glands, biliary duct, lungs, and renal tubules, among others. Immunization with CA-II or lactoferrininduced systemic lesions such as pancreatitis, sialadenitis, cholangitis, or interstitial nephritis in mice models is similar to human IgG4-RD. ${ }^{71)}$ Amylase $\alpha-2 \mathrm{~A},{ }^{72)}$ heat shock $10 \mathrm{kDa}$ protein $1{ }^{73}$ and Helicobacter pylori ${ }^{74), 75)}$ are also disease-associated antigen candidates. Among the organs involved in IgG4$\mathrm{RD}$, recent studies suggest an extremely high association of pancreatic and biliary lesions. ${ }^{25), 26)}$ Peribiliary glands in the biliary tract and pancreatic duct glands associated with pancreatic ducts in humans are intermingled with small amounts of pancreatic exocrine acini ${ }^{76)}$ and biliary tree-derived stem cells constitute pancreatic organogenesis in mice. ${ }^{77)}$ Thus, Nakanuma et al. proposed a new concept of the "biliary diseases with pancreatic counterparts", ${ }^{76)}$ in which targets of type 1 AIP and IgG4-SC may be periductal glands around the bile and pancreatic ducts. Further studies of the pathophysiology of the biliary tract based on its similarity to pancreatic counterparts are warranted.

Recently, two novel candidates of target antigens related to connective tissues in some patients with type 1 AIP have been reported; one is annexin $12^{78)}$ and the other is laminin 511.79),80) Antiannexin A11 IgG4-antibodies were positive in 9/50 patients, and IgG1-Abs in 7/97 patients, and antilaminin 511 IgG4- and IgG1-antibodies were positive in a half of the patients studied.

Role of $\mathbf{B}$ cells. In addition to steroid and immunomodulators, the B-cell depletion by rituximab, which reduces only IgG4, but not IgG1, IgG2, or IgG3, is useful as a therapeutic strategy in IgG4RD. ${ }^{81), 82)}$ A recent study showed expansion of IgG4 ${ }^{+}$B-cell receptor clones in blood and tissue from patients with active IgG4-cholangiopathy, and disappearance of the clones with corticosteroid treatment. ${ }^{82)}$ A recent study showed that increased CD $19^{+}$CD $24^{\text {high }}$ CD $38^{\text {high }}$ Bregs may suppress the disease activity of type 1 AIP, whereas decreased $\mathrm{CD} 19^{+} \mathrm{CD} 24^{\text {high }} \mathrm{CD} 27^{+}$Bregs might be involved in the development of type 1 AIP. ${ }^{83)}$ These findings suggested that specific B-cell responses may play a pivotal role in the pathogenesis of IgG4-RD.

Th1 and Th2 immune balance. The effector cells in IgG4-RDs are poorly understood. $\mathrm{CD}^{+} \mathrm{T}$ cells differentiate from naïve T-cells (Th0) into Th1, Th2, Th17, and Treg cells. In the livers of IgG4-SC patients, a Th2-type immune reaction ${ }^{59), 63)}$ is induced in addition to Th1 responses. ${ }^{66), 71)}$ Th2 cytokines may be involved in progression of the disease process, especially through the maturation and proliferation of local B-cells and plasmacytes.

Regulatory $\mathbf{T}$ cells. Forkhead box P3 is a member of the forkhead/winged-helix family of transcriptional regulators, and functions as the master regulator in the development and function of CD $4^{+} \mathrm{CD} 25^{+}$Tregs. Forkhead box P3 is classified as a naturally occurring $\mathrm{CD} 4^{+} \mathrm{CD} 25^{+}$Treg (nTregs) that originates in the thymus, whereas adaptive Tregs are induced in the periphery by different antigens. $^{84)}$ In type 1 AIP, circulatory naïve $\left(\mathrm{CD} 45 \mathrm{RA}^{+}\right)$Tregs are significantly decreased in the 
peripheral blood, whereas memory $\left(\mathrm{CD} 45 \mathrm{RA}^{-}\right)$Tregs are significantly increased. ${ }^{60}$ ) In addition, prominent infiltration of Tregs with upregulation of IL-10 is observed in the liver of type 1 AIP and IgG4SC patients. ${ }^{59), 62)}$ These findings suggest that increased memory-Tregs in the periphery and local tissues may be an inhibitory immune response against inflammation, although decreased naïve Tregs may be pathogenic.

\section{Concluding remarks}

Recent advances support the concept of IgG4$\mathrm{RD}$, a unique clinical entity, as a systemic disease, and the classification of two distinctive subtypes of AIP. Type 1 AIP is proposed as a pancreatic manifestation of IgG4-RD and type 2 AIP is associated with GELs and inflammatory bowel disease. To improve the diagnostic accuracy and monitoring of disease activity, novel biomarkers more specific for each subtype of AIP, other than serum IgG4 in type 1 and histological findings in type 2 , should be established in the near future. In relapse or steroid refractory cases of type 1 AIP and IgG4-RD, alternative treatment should be established as well as factors for predicting relapse. In the pathogenesis of AIP/IgG4-RD, multi-pathogenic factors including genetic backgrounds, disease-specific antigens, and the role of IgG4 must be clarified.

\section{Acknowledgment}

This study was partially supported by (1) Grant-in-Aid for Scientific Research (C) of the Ministry of Education, Culture, Sports, Science and Technology of Japan (17877850), (2) the Research Program on Intractable Diseases, from the Ministry of Health, Labour and Welfare of Japan, and (3) the Research Program from the Japan Agency for Medical Research and Development (AMED) (17824893).

\section{References}

1) Okazaki, K., Tomiyama, T., Mitsuyama, T., Sumimoto, K. and Uchida, K. (2014) Diagnosis and classification of autoimmune pancreatitis. Autoimmun. Rev. 13, 451-458.

2) Umehara, H., Okazaki, K., Masaki, Y., Kawano, M., Yamamoto, M., Saeki, T. et al. (2012) A novel clinical entity, IgG4-related disease (IgG4RD): general concept and details. Mod. Rheumatol. 22, $1-14$.

3) Stone, J.H., Khosroshahi, A., Deshpande, V., Chan, J.K., Heathcote, J.G., Aalberse, R. et al. (2012) Recommendations for the nomenclature of IgG4- related disease and its individual organ system manifestations. Arthritis Rheum. 64, 3061-3067.

4) Kawaguchi, K., Koike, M., Tsuruta, K., Okamoto, A., Tabata, I. and Fujita, N. (1991) Lymphoplasmacytic sclerosing pancreatitis with cholangitis: a variant of primary sclerosing cholangitis extensively involving pancreas. Hum. Pathol. 22, 387395.

5) Yoshida, K., Toki, F., Takeuchi, T., Watanabe, S., Shiratori, K. and Hayashi, N. (1995) Chronic pancreatitis caused by an autoimmune abnormality. Proposal of the concept of autoimmune pancreatitis. Dig. Dis. Sci. 40, 1561-1568.

6) Comings, D.E., Skubi, K.B., Van Eyes, J. and Motulsky, A.G. (1967) Familial multifocal fibrosclerosis. Ann. Intern. Med. 66, 884-892.

7) Hamano, H., Kawa, S., Horiuchi, A., Unno, H., Furuya, N., Akamatsu, T. et al. (2001) High serum IgG4 concentrations in patients with sclerosing pancreatitis. N. Engl. J. Med. 344, 732-738.

8) Kamisawa, T. and Okamoto, A. (2006) Autoimmune pancreatitis: proposal of IgG4-related sclerosing disease. J. Gastroenterol. 41, 613-625.

9) Shimosegawa, T., Chari, S.T., Frulloni, L., Kamisawa, T., Kawa, S., Mino-Kenudson, M. et al. (2011) International consensus diagnostic criteria for autoimmune pancreatitis: guidelines of the international association of pancreatology. Pancreas 40, 352-358.

10) Yamamoto, M., Takahashi, H., Ohara, M., Suzuki, C., Naishiro, Y., Yamamoto, H. et al. (2006) A new conceptualization for Mikulicz's disease as an IgG4-related plasmacytic disease. Mod. Rheumatol. 16, 335-340.

11) Masaki, Y., Dong, L., Kurose, N., Kitagawa, K., Morikawa, Y., Yamamoto, M. et al. (2009) Proposal for a new clinical entity, IgG4-positive multi-organ lymphoproliferative syndrome: Analysis of 64 cases of IgG4-related disorders. Ann. Rheum. Dis. 68, 1310-1315.

12) Umehara, H., Okazaki, K., Masaki, Y., Kawano, M., Yamamoto, M., Saeki, T. et al. (2012) Comprehensive diagnostic criteria for IgG4-related disease (IgG4-RD), 2011. Mod. Rheumatol. 22, 21-30.

13) Deshpande, V., Zen, Y., Chan, J.K., Yi, E.E., Sato, Y., Yoshino, T. et al. (2012) Consensus statement on the pathology of IgG4-related disease. Mod. Pathol. 25, 1181-1192.

14) Notohara, K., Burgart, L.J., Yadav, D., Chari, S. and Smyrk, T.C. (2003) Idiopathic chronic pancreatitis with periductal lymphoplasmacytic infiltration: clinicopathologic features of 35 cases. Am. J. Surg. Pathol. 27, 1119-1127.

15) Zamboni, G., Luttges, J., Capelli, P., Frulloni, L., Cavallini, G., Pederzoli, P. et al. (2004) Histopathological features of diagnostic and clinical relevance in autoimmune pancreatitis: a study on 53 resection specimens and 9 biopsy specimens. Virchows Arch. 445, 552-563.

16) Masaki, Y., Sugai, S. and Umehara, H. (2010) IgG4related diseases including Mikulicz's disease and sclerosing pancreatitis: diagnostic insights. J. 
Rheumatol. 37, 1380-1385.

17) Kawano, M., Saeki, T., Nakashima, H., Nishi, S., Yamaguchi, Y., Hisano, S. et al. (2010) Proposal for diagnostic criteria for IgG4-related kidney disease. Clin. Exp. Nephrol. 15, 615-626.

18) Okazaki, K. and Umehara, H. (2012) Are classification criteria for IgG4-RD now possible? The concept of IgG4-related disease and proposal of comprehensive diagnostic criteria in Japan. Int. J. Rheumatol. 2012, 357071.

19) Khosroshahi, A., Wallace, Z.S., Crowe, J.L., Akamizu, T., Azumi, A., Carruthers, M.N. et al. (2015) Second international symposium on IgG4related disease. International consensus guidance statement on the management and treatment of IgG4-related disease. Arthritis Rheumatol. 67, 688-699.

20) Shirakashi, M., Yoshifuji, H., Kodama, Y., Chiba, T., Yamamoto, M., Takahashi, H. et al. (2018) Factors in glucocorticoid regimens associated with treatment response and relapses of IgG4-related disease: a multicentre study. Sci. Rep. 8, 10262.

21) Okazaki, K., Chari, S.T., Frulloni, L., Lerch, M.M., Kamisawa, T., Kawa, S. et al. (2017) International consensus for the treatment of autoimmune pancreatitis. Pancreatology 17, 1-6.

22) Hart, P.A., Kamisawa, T., Brugge, W.R., Chung, J.B., Culver, E.L., Czakó, L. et al. (2013) Longterm outcomes of autoimmune pancreatitis: a multicentre, international analysis. Gut $\mathbf{6 2}$, $1771-1776$.

23) Kamisawa, T., Okazaki, K., Kawa, S., Ito, T., Inui, K., Irie, H. et al. (2014) Amendment of the Japanese Consensus Guidelines for Autoimmune Pancreatitis, 2013 III. Treatment and prognosis of autoimmune pancreatitis. J. Gastroenterol. 49, 961-970.

24) Sah, R.P., Chari, S.T., Pannala, R., Sugumar, A., Clain, J.E., Levy, M.J. et al. (2010) Differences in clinical profile and relapse rate of type 1 versus type 2 autoimmune pancreatitis. Gastroenterology 139, 140-148.

25) Hart, P.A., Topazian, M.D., Witzig, T.E., Clain, J.E., Gleeson, F.C., Klebig, R.R. et al. (2013) Treatment of relapsing autoimmune pancreatitis with immunomodulators and rituximab: the Mayo Clinic experience. Gut 62, 1607-1615.

26) Bi, Y., Hart, P.A., Law, R., Clain, J.E., Farnell, M.B., Gleeson, F.C. et al. (2016) Obstructive jaundice in autoimmune pancreatitis can be safely treated with corticosteroids alone without biliary stenting. Pancreatology 16, 391-396.

27) Kamisawa, T., Shimosegawa, T., Okazaki, K., Nishino, T., Watanabe, H., Kanno, A. et al. (2009) Standard steroid therapy for autoimmune pancreatitis. Gut 58, 1504-1507.

28) Nishimori, I., Tamakoshi, A. and Otsuki, M. (2007) Prevalence of autoimmune pancreatitis in Japan from a nationwide survey in 2002. J. Gastroenterol. 42 (suppl), 6-8.

29) Ito, T., Nishimori, I., Inoue, N., Kawabe, K., Gibo, J., Arita, Y. et al. (2007) Treatment for auto- immune pancreatitis: consensus on the treatment for patients with autoimmune pancreatitis in Japan. J. Gastroenterol. 42 (suppl), 50-58.

30) Muraki, T., Hamano, H., Ochi, Y., Komatsu, K., Komiyama, Y., Arakura, N. et al. (2006) Autoimmune pancreatitis and complement activation system. Pancreas 32, 16-21.

31) Saeki, T., Kawano, M., Mizushima, I., Yamamoto, M., Wada, Y., Nakashima, H. et al. (2013) The clinical course of patients with IgG4-related kidney disease. Kidney Int. 84, 826-833.

32) Park, D.H., Kim, M.H., Oh, H.B., Kwon, O.J., Choi, Y.J., Lee, S.S. et al. (2008) Substitution of aspartic acid at position 57 of the DQb1 affects relapse of autoimmune pancreatitis. Gastroenterology 134, 440-446.

33) Masamune, A., Nishimori, I., Kikuta, K., Tsuji, I. Mizuno, N., Iiyama, T. et al. (2017) Randomised controlled trial of long-term maintenance corticosteroid therapy in patients with autoimmune pancreatitis. Gut 66, 487-494.

34) Ko, S.B., Mizuno, N., Yatabe, Y., Yoshikawa, T., Ishiguro, H., Yamamoto, A. et al. (2010) Corticosteroids correct aberrant CFTR localization in the duct and regenerate acinar cells in autoimmune pancreatitis. Gastroenterology 138, 1988-1996.

35) Takuma, K., Kamisawa, T., Tabata, T., Inaba, Y., Egawa, N. and Igarashi, Y. (2011) Short-term and long-term outcomes of autoimmune pancreatitis. Eur. J. Gastroenterol. Hepatol. 23, 146-152.

36) Uchida, K., Yazumi, S., Nishio, A., Kusuda, T., Koyabu, M., Fukata, M. et al. (2009) Long-term outcome of autoimmune pancreatitis. J. Gastroenterol. 44, 726-732.

37) Frulloni, L., Scattolini, C., Falconi, M., Zamboni, G., Capelli, P., Manfredi, R. et al. (2009) Autoimmune pancreatitis: differences between the focal and diffuse forms in 87 patients. Am. J. Gastroenterol. 104, 2288-2294.

38) Maruyama, M., Arakura, N., Ozaki, Y., Watanabe, T., Ito, T., Yoneda, S. et al. (2013) Type 1 autoimmune pancreatitis can transform into chronic pancreatitis: a long-term follow-up study of 73 Japanese patients. Int. J. Rheumatol. 2013, 272595 .

39) Maruyama, M., Watanabe, T., Kanai, K., Oguchi, T., Asano, J., Ito, T. et al. (2014) Autoimmune pancreatitis can develop into chronic pancreatitis. Orphanet J. Rare Dis. 9, 77.

40) Ikeura, T., Miyoshi, H., Uchida, K., Fukui, T., Shimatani, M., Fukui, Y. et al. (2014) Relationship between autoimmune pancreatitis and pancreatic cancer: a single-center experience. Pancreatology 14, 373-379.

41) Hirano, K., Tada, M., Sasahira, N., Isayama, H., Mizuno, S., Takagi, K. et al. (2014) Incidence of malignancies in patients with IgG4-related disease. Intern. Med. 53, 171-176.

42) Shiokawa, M., Kodama, Y., Yoshimura, K., Kawanami, C., Mimura, J., Yamashita, Y. et al. (2013) Risk of cancer in patients with autoimmune pancreatitis. Am. J. Gastroenterol. 108, 610-617. 
43) Yamamoto, M., Takahashi, H., Tabeya, T., Suzuki, C., Naishiro, Y., Ishigami, K. et al. (2012) Risk of malignancies in IgG4-related disease. Mod. Rheumatol. 22, 414-418.

44) Kamisawa, T., Tsuruta, K., Okamoto, A., Horiguchi, S., Hayashi, Y., Yun, X. et al. (2009) Frequent and significant K-ras mutation in the pancreas, the bile duct, and the gallbladder in autoimmune pancreatitis. Pancreas 38, 890-895.

45) Okazaki, K. and Uchida, K. (2015) Autoimmune pancreatitis: the past, present, and future. Pancreas 44, 1006-1016.

46) Kawa, S., Ota, M., Yoshizawa, K., Horiuchi, A., Hamano, H., Ochi, Y. et al. (2002) HLA DRB10405-DQB10401 haplotype is associated with autoimmune pancreatitis in the Japanese population. Gastroenterology 122, 1264-1269.

47) Umemura, T., Ota, M., Hamano, H., Katsuyama, Y., Kiyosawa, K. and Kawa, S. (2006) Genetic association of $\mathrm{Fc}$ receptor-like 3 polymorphisms with autoimmune pancreatitis in Japanese patients. Gut 55, 1367-1368.

48) Umemura, T., Katsuyama, Y., Hamano, H., Kitahara, K., Takayama, M., Arakura, N. et al. (2009) Association analysis of Toll-like receptor 4 polymorphisms with autoimmune pancreatitis. Hum. Immunol. 70, 742-746.

49) Chang, M.C., Chang, Y.T., Tien, Y.W., Liang, P.C., Jan, I.S., Wei, S.C. et al. (2007) T-cell regulatory gene CTLA-4 polymorphism/haplotype association with autoimmune pancreatitis. Clin. Chem. 53, 1700-1705.

50) Watanabe, T., Yamashita, K., Fujikawa, S., Sakurai, T., Kudo, M., Shiokawa, M. et al. (2012) Involvement of activation of Toll-like receptors and nucleotide-binding oligomerization domain-like receptors in enhanced IgG4 responses in autoimmune pancreatitis. Arthritis Rheum. 64, 914-924.

51) Watanabe, T., Yamashita, K., Sakurai, T., Kudo, M., Shiokawa, M., Uza, N. et al. (2013) Toll-like receptor activation in basophils contributes to the development of IgG4 related disease. J. Gastroenterol. 48, 247-253.

52) Fukui, Y., Uchida, K., Sakaguchi, Y., Fukui, T., Nishio, A., Shikata, N. et al. (2015) Possible involvement of Toll-like receptor 7 in the development of type 1 autoimmune pancreatitis. J. Gastroenterol. 50, 435-444.

53) Yamashina, M., Nishio, A., Nakayama, S., Okazaki, T., Uchida, K., Fukui, T. et al. (2012) Comparative study on experimental autoimmune pancreatitis and its extrapancreatic involvement in mice. Pancreas 41, 1255-1262.

54) Yanagawa, M., Uchida, K., Ando, Y., Tomiyama, T., Yamaguchi, T., Ikeura, T. et al. (2018) Basophils activated via TLR signaling may contribute to pathophysiology of type 1 autoimmune pancreatitis. J. Gastroenterol. 53, 449-460.

55) Van der Neut Kolfschoten, M., Schuurman, J., Losen, M., Bleeker, W.K., Martínez-Martínez, P., Vermeulen, E. et al. (2007) Anti-inflammatory activity of human IgG4 antibodies by dynamic Fab arm exchange. Science 317, 1554-1557.

56) Kawa, S., Kitahara, K., Hamano, H., Ozaki, Y., Arakura, N., Yoshizawa, K. et al. (2008) A novel immunoglobulin-immunoglobulin interaction in autoimmunity. PLoS One 3, e1637.

57) Ishii, K., Amagai, M., Hall, R.P., Hashimoto, T., Takayanagi, A., Gamou, S. et al. (1997) Characterization of autoantibodies in pemphigus using antigen-specific enzyme-linked immunosorbent assays with baculovirus-expressed recombinant desmogleins. J. Immunol. 159, 2010-2017.

58) Davies, A.M., Rispens, T., Ooijevaar-de Heer, P., Gould, H.J., Jefferis, R., Aalberse, R.C. et al. (2014) Structural determinants of unique properties of human IgG4-Fc. J. Mol. Biol. 426, 630-644.

59) Zen, Y., Fujii, T., Harada, K., Kawano, M., Yamada, K., Takahira, M. et al. (2007) Th2 and regulatory immune reactions are increased in immunoglobin G4-related sclerosing pancreatitis and cholangitis. Hepatology 45, 1538-1546.

60) Miyoshi, H., Uchida, K., Taniguchi, T., Yazumi, S. Matsushita, M., Takaoka, M. et al. (2008) Circulating naïve and $\mathrm{CD} 4^{+} \mathrm{CD} 25^{\text {high }}$ regulatory $\mathrm{T}$ cells in patients with autoimmune pancreatitis. Pancreas 36, 133-140.

61) Koyabu, M., Uchida, K., Miyoshi, H., Sakaguchi, Y., Fukui, T., Ikeda, H. et al. (2010) Analysis of regulatory $\mathrm{T}$ cells and IgG4-positive plasma cells among patients of IgG4-related sclerosing cholangitis and autoimmune liver diseases. J. Gastroenterol. 45, 732-741.

62) Kusuda, T., Uchida, K., Miyoshi, H., Koyabu, M., Satoi, S., Takaoka, M. et al. (2011) Involvement of inducible costimulator- and interleukin 10-positive regulatory $\mathrm{T}$ cells in the development of $\mathrm{IgG4}$ related autoimmune pancreatitis. Pancreas 40, $1120-1130$

63) Tanaka, A., Moriyama, M., Nakashima, H., Miyake, K., Hayashida, J.N., Maehara, T. et al. (2012) Th2 and regulatory immune reactions contribute to IgG4 production and the initiation of Mikulicz disease. Arthritis Rheum. 64, 254-263.

64) Muraki, T., Hamano, H., Ochi, Y., Komatsu, K., Komiyama, Y., Arakura, N. et al. (2006) Autoimmune pancreatitis and complement activation system. Pancreas 32, 16-21.

65) Uchida, K., Okazaki, K., Konishi, Y., Ohana, M., Takakuwa, H., Hajiro, K. et al. (2000) Clinical analysis of autoimmune-related pancreatitis. Am. J. Gastroenterol. 95, 2788-2794.

66) Okazaki, K., Uchida, K., Ohana, M., Nakase, H., Uose, S., Inai, M. et al. (2000) Autoimmunerelated pancreatitis is associated with autoantibodies and a Th1/Th2-type cellular immune response. Gastroenterology 118, 573-581.

67) Nishi, H., Tojo, A., Onozato, M.L., Jimbo, R., Nangaku, M., Uozaki, H. et al. (2007) Anticarbonic anhydrase II antibody in autoimmune pancreatitis and tubulointerstitial nephritis. Nephrol. Dial. Transplant. 22, 1273-1275.

68) Aparisi, L., Farre, A., Gomez-Cambronero, L., Martinez, J., De Las Heras, G., Corts, J. et al. 
(2005) Antibodies to carbonic anhydrase and IgG4 levels in idiopathic chronic pancreatitis: relevance for diagnosis of autoimmune pancreatitis. Gut 54, 703-709.

69) Nishimori, I., Miyaji, E., Morimoto, K., Nagao, K., Kamada, M. and Onishi, S. (2005) Serum antibodies to carbonic anhydrase IV in patients with autoimmune pancreatitis. Gut 54, 274-281.

70) Asada, M., Nishio, A., Uchida, K., Kido, M., Ueno, S., Uza, N. et al. (2006) Identification of a novel autoantibody against pancreatic secretory trypsin inhibitor in patients with autoimmune pancreatitis. Pancreas 33, 20-26.

71) Uchida, K., Okazaki, K., Nishi, T., Uose, S., Nakase, H., Ohana, M. et al. (2002) Experimental immunemediated pancreatitis in neonatally thymectomized mice immunized with carbonic anhydrase II and lactoferrin. Lab. Invest. 82, 411-424.

72) Endo, T., Takizawa, S., Tanaka, S., Takahashi, M., Fujii, H., Kamisawa, T. et al. (2009) Amylase $\alpha$-2A autoantibodies: novel marker of autoimmune pancreatitis and fulminant type 1 diabetes. Diabetes $\mathbf{5 8}, 732-737$.

73) Takizawa, S., Endo, T., Wanjia, X., Tanaka, S., Takahashi, M. and Kobayashi, T. (2009) HSP 10 is a new autoantigen in both autoimmune pancreatitis and fulminant type 1 diabetes. Biochem. Biophys. Res. Commun. 386, 192-196.

74) Kountouras, J., Zavos, C., Gavalas, E. and Tzilves, D. (2007) Challenge in the pathogenesis of autoimmune pancreatitis: potential role of Helicobacter pylori infection via molecular mimicry. Gastroenterology 133, 368-369.

75) Kountouras, J., Zavos, C. and Chatzopoulos, D. (2005) A concept on the role of Helicobacter pylori infection in autoimmune pancreatitis. J. Cell Mol. Med. 9, 196-207.

76) Nakanuma, Y. (2010) A novel approach to biliary tract pathology based on similarities to pancreatic counterparts: is the biliary tract an incomplete pancreas? Pathol. Int. 60, 419-429.

77) Wang, Y., Lanzoni, G., Carpino, G., Cui, C.B., Dominguez-Bendala, J., Wauthier, E. et al. (2013)
Biliary tree stem cells, precursors to pancreatic committed progenitors: evidence for possible lifelong pancreatic organogenesis. Stem Cells 31, 1966-1979.

78) Hubers, L.M., Vos, H., Schuurman, A.R., Erken, R., Oude Elferink, R.P., Burgering, B. et al. (2018) Annexin A11 is targeted by IgG4 and IgG1 autoantibodies in IgG4-related disease. Gut 67, $728-735$

79) Shiokawa, M., Kodama, Y., Kuriyama, K., Yoshimura, K., Tomono, T., Morita, T. et al. (2016) Pathogenicity of IgG in patients with IgG4related disease. Gut 65, 1322-1332.

80) Shiokawa, M., Kodama, Y., Sekiguchi, K., Kuwada, T., Tomono, T., Kuriyama, K. et al. (2018) Laminin 511 is a target antigen in autoimmune pancreatitis. Sci. Transl. Med. 10, eaaq0997.

81) Ghazale, A., Chari, S.T., Zhang, L., Smyrk, T.C., Takahashi, N., Levy, M.J. et al. (2008) Immunoglobulin G4-associated cholangitis: clinical profile and response to therapy. Gastroenterology 134, 706-715.

82) Maillette de Buy Wenniger, L.J., Doorenspleet, M.E., Klarenbeek, P.L., Verheij, J., Baas, F., Elferink, R.P. et al. (2013) Immunoglobulin G4+ clones identified by next-generation sequencing dominate the $\mathrm{B}$ cell receptor repertoire in immunoglobulin G4 associated cholangitis. Hepatology 57, 2390-2398.

83) Sumimoto, K., Uchida, K., Kusuda, T., Mitsuyama, T., Sakaguchi, Y., Fukui, T. et al. (2014) The role of $\mathrm{CD} 19^{+} \mathrm{CD} 24^{\text {high }} \mathrm{CD} 38^{\text {high }}$ and $\mathrm{CD} 19^{+} \mathrm{CD} 24^{\text {high }} \mathrm{CD} 27^{+}$regulatory $\mathrm{B}$ cells in patients with type 1 autoimmune pancreatitis. Pancreatology 14, 193-200.

84) Valencia, X. and Lipsky, P.E. (2007) $\mathrm{CD} 4{ }^{+} \mathrm{CD} 25^{+} \mathrm{FoxP}^{+}$regulatory $\mathrm{T}$ cells in autoimmune diseases. Nat. Clin. Pract. Rheumatol. 3, 619-626.

(Received Aug. 27, 2018; accepted Oct. 10, 2018) 


\section{Profile}

Kazuichi Okazaki was born in Kochi Prefecture, Japan, and has been working in the field of gastroenterology and clinical immunology after he graduated from Kyoto University. After 5 years' clinical training and the award of his Ph.D. thesis, he started his academic career as an Assistant Professor at the School of Medicine, Kochi University, Japan. After 2 years as a visiting researcher at New York Medical College and the University of Medicine and Dentist of New Jersey, he was appointed as an Associate Professor, Kochi University in 1994. In 1996, he returned to the Department of Gastroenterology, Kyoto University, as an Associate Professor. In 2003, he was nominated as Chairman and Professor of the Department of Gastroenterology and Hepatology, Kansai Medical University, Osaka. His main academic interest is in

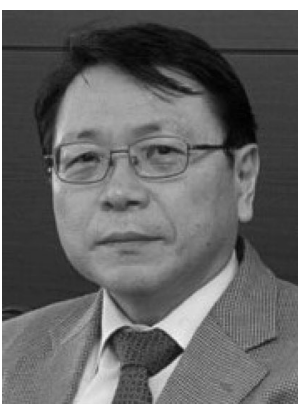
pancreatology and clinical immunology, especially autoimmune pancreatitis (AIP) as an IgG4-related disease, and inflammatory bowel disease. He has published more than 470 English-language articles in the field of gastroenterology. He was appointed as President of the Japan Pancreas Society (JPS) and has been councilor of the International Association of Pancreatology (IAP), Asian Pacific Association of Gastroenterology (APAGE), the Organization of Japanese Digestive Disease Week (JDDW), and Japanese Society of Gastroenterology (JSGE). He organized the committee for the Japanese Diagnostic Criteria for AIP in 2006 supported by the JPS and contributed to the International Consensus Diagnostic Criteria for AIP in 2010 supported by the IAP. He has recently organized nationwide study groups for IgG4-related disease (2010-2013, 2017-2019) supported by the Ministry of Health, Labour and Welfare of Japan. He has been invited as a guest speaker at meetings held in Asia (Korea, Thailand, Taipei), Oceania (Australia), Europe (Italy) and the United States (APA, AGA). 